\title{
ALMA OBSERVATIONS OF THE COLDEST PLACE IN THE UNIVERSE: THE BOOMERANG NEBULA
}

\author{
R. SAHAi ${ }^{1}$, W. H. T. Vlemmings ${ }^{2}$, P. J. HugGins ${ }^{3}$, L.-Å. NyMAN ${ }^{4,5}$, AND I. Gonidakis ${ }^{6}$ \\ ${ }^{1}$ Jet Propulsion Laboratory, MS 183-900, California Institute of Technology, Pasadena, CA 91109, USA; raghvendra.sahai@jpl.nasa.gov \\ ${ }^{2}$ Department of Earth and Space Sciences, Chalmers University of Technology, Onsala Space Observatory, SE-43992 Onsala, Sweden \\ ${ }^{3}$ Physics Department, New York University, 4 Washington Place, New York, NY 10003, USA \\ ${ }^{4}$ Joint ALMA Observatory (JAO), Alonso de Cordova 3107, Vitacura, Santiago de Chile, Chile \\ ${ }^{5}$ European Southern Observatory, Alonso de Cordova 3107, Vitacura, Santiago, Chile \\ ${ }^{6}$ CSIRO Astronomy and Space Science, Australia Telescope National Facility, Marsfield NSW 2122, Australia \\ Received 2013 July 2; accepted 2013 August 19; published 2013 October 18
}

\begin{abstract}
The Boomerang Nebula is the coldest known object in the universe, and an extreme member of the class of pre-planetary nebulae, objects which represent a short-lived transitional phase between the asymptotic giant branch and planetary nebula evolutionary stages. Previous single-dish CO $(J=1-0)$ observations (with a $45^{\prime \prime}$ beam) showed that the high-speed outflow in this object has cooled to a temperature significantly below the temperature of the cosmic background radiation. Here we report the first observations of the Boomerang Nebula with ALMA in the $\mathrm{CO} J=2-1$ and $J=1-0$ lines to resolve the structure of this ultra-cold nebula. We find a central hourglass-shaped nebula surrounded by a patchy, but roughly round, cold high-velocity outflow. We compare the ALMA data with visible-light images obtained with the Hubble Space Telescope and confirm that the limb-brightened bipolar lobes seen in these data represent hollow cavities with dense walls of molecular gas and dust producing both the molecular-emission-line and scattered-light structures seen at millimeter and visible wavelengths. The large diffuse biconical shape of the nebula seen in the visible wavelength range is likely due to preferential illumination of the cold, high-velocity outflow. We find a compact source of millimeter-wave continuum in the nebular waist - these data, together with sensitive upper limits on the radio continuum using observations with ATCA, indicate the presence of a substantial mass of very large (millimeter-sized) grains in the waist of the nebula. Another unanticipated result is the detection of $\mathrm{CO}$ emission regions beyond the ultra-cold region which indicate the re-warming of the cold gas, most likely due to photoelectric grain heating.
\end{abstract}

Key words: circumstellar matter - planetary nebulae: individual (Boomerang Nebula) - radio lines: stars - stars: AGB and post-AGB - stars: mass-loss - stars: winds, outflows

Online-only material: color figures

\section{INTRODUCTION}

The Boomerang Nebula, discovered by Wegner \& Glass (1979), holds the distinction of being the coldest known object in the universe (Sahai \& Nyman 1997, hereafter SN97). It is a bipolar pre-planetary nebula (PPN), representing a short-lived $(\sim 1000 \mathrm{yr})$ transition phase during which asymptotic giant branch (AGB) stars and their round circumstellar envelopes evolve into planetary nebulae with a breathtaking variety of aspherical geometrical shapes and symmetries (e.g., Sahai et al. 2011b). The Boomerang's estimated prodigious massloss rate $\left(0.001 M_{\odot} \mathrm{yr}^{-1}\right)$ and low-luminosity $\left(300 L_{\odot}\right)$ lack an explanation in terms of current paradigms for dusty mass loss and the standard evolutionary theory of intermediate-mass stars.

Single-dish CO $(J=1-0)$ observations (SN97) showed an extended high-speed outflow in absorption against the microwave background, implying that the nebula has cooled to a temperature significantly below that of the cosmic background radiation $\left(T_{\mathrm{cmb}}=2.7 \mathrm{~K}\right)$ due to adiabatic expansion. Like all PPNs imaged at visible wavelengths at high-resolution (e.g., Sahai et al. 2007), the Boomerang appears aspherical, with an hourglass morphology as seen in the light reflected by dust grains (SN97; also see Hubble Heritage Release STScI-2005-257). However, the low angular resolution of the CO observations (45" and $24^{\prime \prime}$ at $J=1-0$ and $J=2-1$, respectively) did not show strong departures from sphericity, bringing up fundamental questions about

\footnotetext{
7 http://hubblesite.org/newscenter/archive/releases/2005/25/image/b/
}

the relationship between the molecular outflow and the dusty nebula, and thus about its very formation. High angular resolution mapping of the molecular gas distribution in the Boomerang was needed to resolve the apparent discrepancy between the molecular and visible-light morphology. This paper reports our mapping of this ultra-cold nebula with ALMA in Cycle 0 (using the compact configuration) with $\sim 2^{\prime \prime}-4^{\prime \prime}$ resolution, in the CO $J=2-1$ and $J=1-0$ lines, as well as millimeter-wave continuum emission at 1.3 and $2.6 \mathrm{~mm}$. We compare these high-resolution images of the molecular gas with archival visible-light images obtained with the Hubble Space Telescope (HST). We also report radio continuum observations with the Australia Telescope Compact Array (ATCA), which we obtained in order to help our understanding of the millimeter-wave continuum.

The outline of the paper is as follows. In Section 2 we describe the observational setups, the data reduction, and calibration procedures. In Section 3 we present our main observational results as derived from the visible-light data (Section 3.1), the $\mathrm{CO}(J=2-1)$ and $\mathrm{CO}(J=1-0)$ line data (Sections 3.2 and 3.3), and the continuum data (Section 3.4). In Section 4, we discuss and analyze these results, and in Section 5 we present the main conclusions of our study. We adopt a value of $1.5 \mathrm{kpc}$ for the distance to the Boomerang (as inferred by SN97).

\section{OBSERVATIONS}

The CO $(J=1-0)$ emission of the Boomerang Nebula, at a rest frequency of $115.2712 \mathrm{GHz}$ and the nearby continuum 
were observed using ALMA band 3 on 2011 November 29 and December 31. The CO $(J=2-1)$ emission line at $230.3581 \mathrm{GHz}$ and the nearby continuum were observed using ALMA band 6 on 2011 November 28. In both cases, the data contain four spectral windows of $1.875 \mathrm{GHz}$ width and with 3840 channels. The band 3 observations have spectral windows centered on approximately 100.7, 102.6, 112.7, and $114.6 \mathrm{GHz}$ while the band 6 observations have windows centered on 228.4, 230.4, 242.4 and $244.4 \mathrm{GHz}$. The channel spacing of $0.488 \mathrm{MHz}$ corresponds to $1.27 \mathrm{~km} \mathrm{~s}^{-1}$ for the CO $(J=1-0)$ and $0.63 \mathrm{~km} \mathrm{~s}^{-1}$ for the $\mathrm{CO}(J=2-1)$ line. The data were taken using the compact ALMA Cycle 0 configuration, with baselines ranging from $14 \mathrm{~m}$ up to $200 \mathrm{~m}$.

In the case of the $\mathrm{CO}(J=1-0)$ observations, a 7-point mosaic, using 15 antennas in November and 18 antennas in December, was used to map a circular area with a radius of $\sim 00^{\prime} .9$ centered on R.A. $=12^{\mathrm{h}} 44^{\mathrm{m}} 45^{\mathrm{s}} .449$ and decl. $=-54^{\circ} 31^{\prime} 11^{\prime \prime} .388$. The CO $(J=2-1)$ was observed with a single pointing at the central position using 15 antennas, 2 of which were flagged due to problems with the water vapor radiometer (WVR). The pointing position corresponds to the Simbad coordinates of the Boomerang Nebula, R.A. $=12^{\mathrm{h}} 44^{\mathrm{m}} 45^{\mathrm{s}} .45$ and decl. $=$ $-54^{\circ} 31^{\prime} 11^{\prime \prime}$. 4 . The total observing time for the $\mathrm{CO}(J=1-0)$ line was $3.25 \mathrm{hr}$ with each mosaic pointing observed for $\sim 19$ minutes in total. The single pointing of the $\mathrm{CO}(J=2-1)$ line was observed for only 6 minutes. In both cases, bandpass and gain calibration were performed on the quasar J1329-5608, while flux calibration was done using Mars. The flux calibration was bootstrapped between spectral windows affected by line emission from Mars itself to the gain calibrator. We measured a flux of $1.63 \pm 0.02 \mathrm{Jy}$ beam $^{-1}$ for $\mathrm{J} 1329-5608$ in November in band 3, which increased to $1.80 \pm 0.03 \mathrm{Jy}$ beam $^{-1}$ in December. In band 6 , we measured a flux of $1.04 \pm 0.04 \mathrm{Jy} \mathrm{beam}^{-1}$. We estimate the final absolute flux calibration to be accurate to within $3 \%$ in band 3 and $5 \%$ in band 6 .

The data were reduced using the Common Astronomy Software Application (CASA 3.4.0). After corrections for time and frequency dependence of the system temperatures and rapid atmospheric variations at each antenna using WVR data, we improved the antenna positions. Subsequently, bandpass and gain calibration were done, and the calibration solutions determined on J1329-5608 were applied to the Boomerang Nebula. Imaging was done using the CASA clean algorithm after a continuum subtraction was performed on the emission line data. As a significant amount of flux of the $\mathrm{CO}(J=1-0)$ line was resolved out, we performed various cleaning rounds using different weighing schemes (from natural to uniform) and different data tapering to assess the robustness of the observed structure. As consistent structure was recovered in the different cleaning runs, we conclude that these are not artifacts of the missing short spacings. Our final CO $(J=1-0)$ image was created by channel averaging to a spectral resolution of $6.25 \mathrm{~km} \mathrm{~s}^{-1}$, using natural weighing, a Gaussian taper of $50 \mathrm{k} \lambda$, and a restoring beam of $4^{\prime \prime} .5 \times 3^{\prime \prime} .0$ at a position angle of $-30^{\circ}$. In the line-free channels, the resulting rms noise was $\sim 2.5 \mathrm{mJy}^{\text {beam }^{-1}}$. The CO $(J=$ 2-1) map was imaged at a spectral resolution of $0.63 \mathrm{~km} \mathrm{~s}^{-1}$, Briggs weighing, and a Gaussian taper of $100 \mathrm{k} \lambda$; the beam was 2 . $40 \times 1$ 1.55 at a position angle of -3.5 . In the line-free channels, this resulted in an rms noise of $\sim 7.5 \mathrm{mJy}^{\text {beam }^{-1}}$. Finally, we combined a $\mathrm{CO}(J=1-0)$ image cube with a spectral resolution of $8 \mathrm{~km} \mathrm{~s}^{-1}$ with the original SEST observations (SN97) using the CASA task feather, in which the regridded image data is combined in the Fourier plane. A lower spectral resolution was used to reduce the noise contribution from the SEST observations.

HST images, taken with the ACS/HRC and WFPC2 instruments, through the F606W wide-band filter, on 2005 March 25, and 1998 March 23 via programs GO 10378 and GO 6856, were extracted from the MAST archive and the Hubble Legacy Archive, respectively. For the HRC data two 160 s exposures were registered and combined in order to remove cosmic-rays. For the WFPC2 data, the Legacy Archive provided an average image combining two $500 \mathrm{~s}$ exposures. Since part of the extended nebula is obscured by the occulting finger of the ACS/HRC coronagraph, we used the WFPC2 image (in which the central region is saturated) to compare the large-scale visible-light morphology with our ALMA data.

We observed the Boomerang Nebula in Director's Discretionary Time with ATCA on 2012 June 21. A $10 \mathrm{hr}$ observation run (project code CX241) was carried out with the 6.0D array configuration, using $2 \mathrm{GHz}$ bandwidths at 5.5, 9, 22, and $24 \mathrm{GHz}$ with the Compact Array Broadband Backend (CABB). The calibration and the imaging of the data were done using the MIRIAD package. Bandpass and flux calibration were performed on the standard calibrator 1934-638 and phase calibration was performed on 1326-5256. The fluxes are in agreement with the values presented in the ATCA calibrators database, within an uncertainty of less than $10 \%$. After calibration, the imaging of the source was performed using multifrequency synthesis with a single continuum image produced for the 22 and $24 \mathrm{GHz}$ bands (identified with the central frequency of $23 \mathrm{GHz}$ ). The beam size of the observations was 2 .' $5 \times 3^{\prime \prime} .1,1^{\prime \prime} .5 \times 1^{\prime \prime} .9$, and $0.6 \times 0.7$ for the 5.5, 9, and $23 \mathrm{GHz}$ observations, respectively.

\section{RESULTS}

\subsection{Visible-light Imaging}

We describe the HST imaging first, as this shows the structural details of the nebula (in scattered light), at the highest angular resolution $(\sim 0$.'05; over-sampled by a factor of two in the HRC with a plate scale of $0^{\prime \prime} .025$ pixel $^{-1}$ ). The HRC image (Figure 1) shows a limb-brightened lobe in the south, which, at its base, flares out from the center in a wide $\mathrm{V}$ shape to an axial distance of 0.7 , and then follows a roughly cylindrical shape, i.e., the western and eastern limbs are parallel, out to about an axial offset of 4".4, beyond which they curve inward and merge; the resulting lobe-end is located at an axial offset of 5".6. The lobe axis is oriented at P.A. $\sim 173^{\circ}$.

The northern lobe, also limb-brightened, is much fainter and has a more complex structure. There is a wide V-shaped flared structure as in the south, which transitions toward a more cylindrical shape, however, the western and eastern limbs of this lobe are not exactly parallel. The average P.A. of the long axis of the $\mathrm{N}$ lobe is about $6^{\circ}$, and thus not aligned with that of the $\mathrm{S}$ lobe. The lateral width of the $\mathrm{N}$ lobe (about 2.5 , measured at an axial offset of $\left.2^{\prime \prime}\right)$ is larger than that of the $S$ lobe $\left(2^{\prime \prime} .1\right)$. The $\mathrm{W}$ and $\mathrm{E}$ limbs of the $\mathrm{N}$ lobe curve inward toward the lobe-axis and merge, and the end of the lobe is located at an axial offset of 4 .'2.

Each limb-brightened lobe appears embedded in a larger, more diffuse conical nebulosity with an opening angle that is the same as that of its wide $\mathrm{V}$-shaped inner region (measuring about $90^{\circ}$ in the north and $100^{\circ}$ in the south).

A bright linear feature can be seen in the middle of the $\mathrm{S}$ lobe, emanating from the central star, extending out to an offset of about $1^{\prime \prime}$, at P.A. $=180^{\circ}$. This feature has significant structure, 


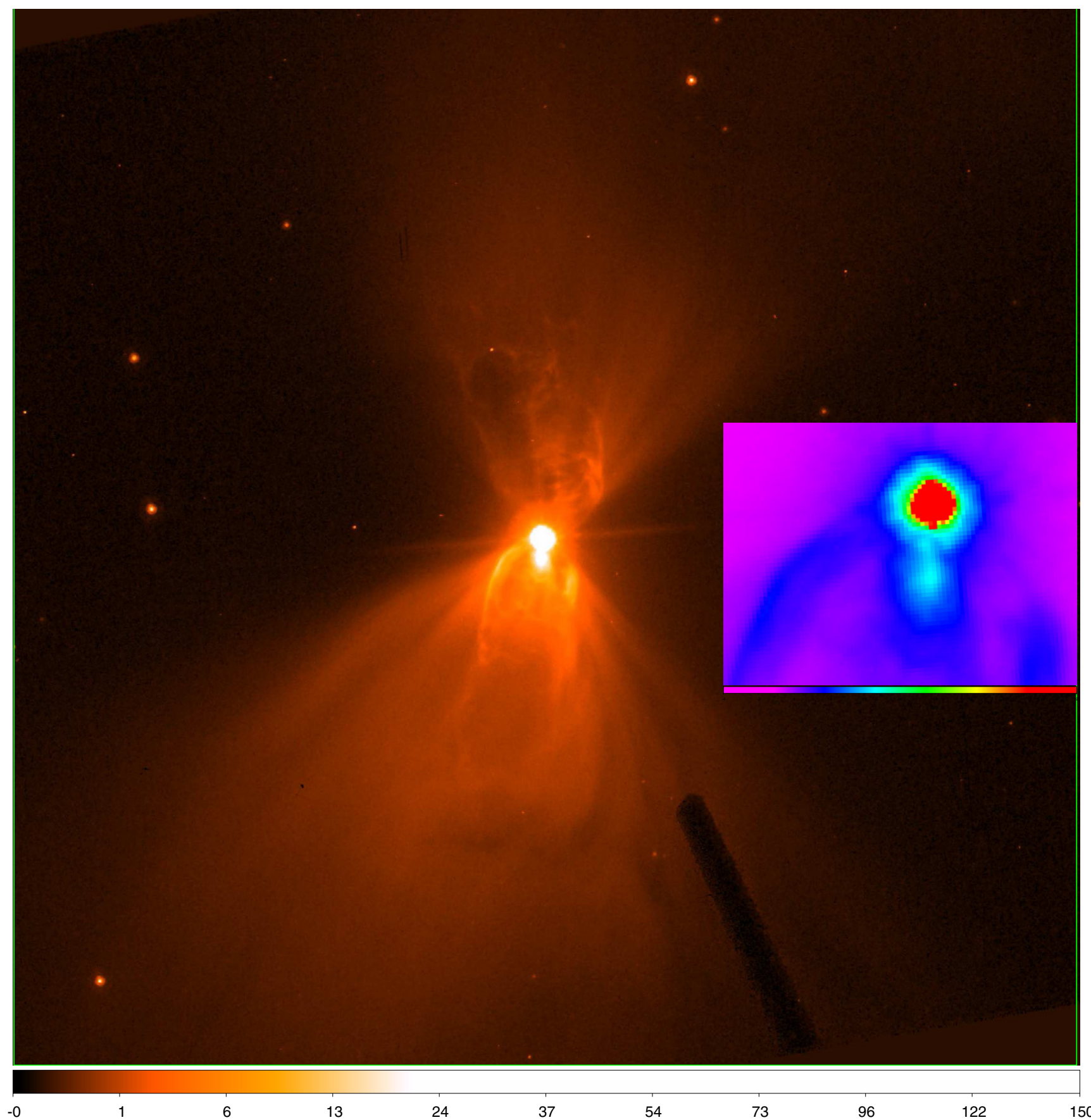

Figure 1. HST F606W image $\left(24^{\prime \prime} \times 24^{\prime \prime}\right.$, shown using a square-root stretch) - the intensity values shown in the scalar-bar at the bottom are in units of $1.268 \times$ $10^{(-19)} \mathrm{erg} \mathrm{s}^{-1} \mathrm{~cm}^{-2} \AA^{-1}$. The inset is a magnified view of the central region, showing the presence of two knots along the central linear feature in the $\mathrm{S}$ lobe. The dark linear feature at the lower right is due to the occulting finger of the ACS/HRC coronagraph.

the most noteworthy being the presence of two bright knots, one at a radial distance, $r=0.43$, P.A. $=179^{\circ}$ from the center, and another slightly fainter and smaller, located at $r=0.23$, P.A. $=172^{\circ}$.

$$
\text { 3.2. } C O(J=2-1)
$$

We show the CO $(J=2-1)$ emission as a function of radial velocity in Figure 2 . At the systemic velocity $\left(\sim-10 \mathrm{~km} \mathrm{~s}^{-1}\right.$; SN97), the CO $(J=2-1)$ image represents a cut of the density structure in the sky plane, and shows a roughly hourglassshaped, bipolar nebula in emission with a central waist. The two limb-brightened lobes defining the hourglass cover a region of about 13". $6 \times$ 4". 4 (see Figure 3(a)); the waist has a lateral extent of about 3 ." 8 (as measured from an intensity cut across the waist between locations where the intensity drops to half its value at the geometrical center of the waist). Spectra extracted from representative locations within the lobes show double-peaked profiles (Figure 3(b)), as expected due to emission from the front and back parts of lobes that are hollow in their interior. The mean velocity for the $\mathrm{S}$ lobe is blue-shifted from that of the $\mathrm{N}$ lobe, implying that the southern (northern) lobe is tilted toward (away from) us. In contrast, the profile toward the center of the nebula, is centrally peaked. The width of the profiles at their base (full width at zero intensity, FWZI) is about $65 \mathrm{~km} \mathrm{~s}^{-1}$.

We do not expect that there is any loss of flux in our CO $(J=2-1)$ image of the Boomerang because the total 


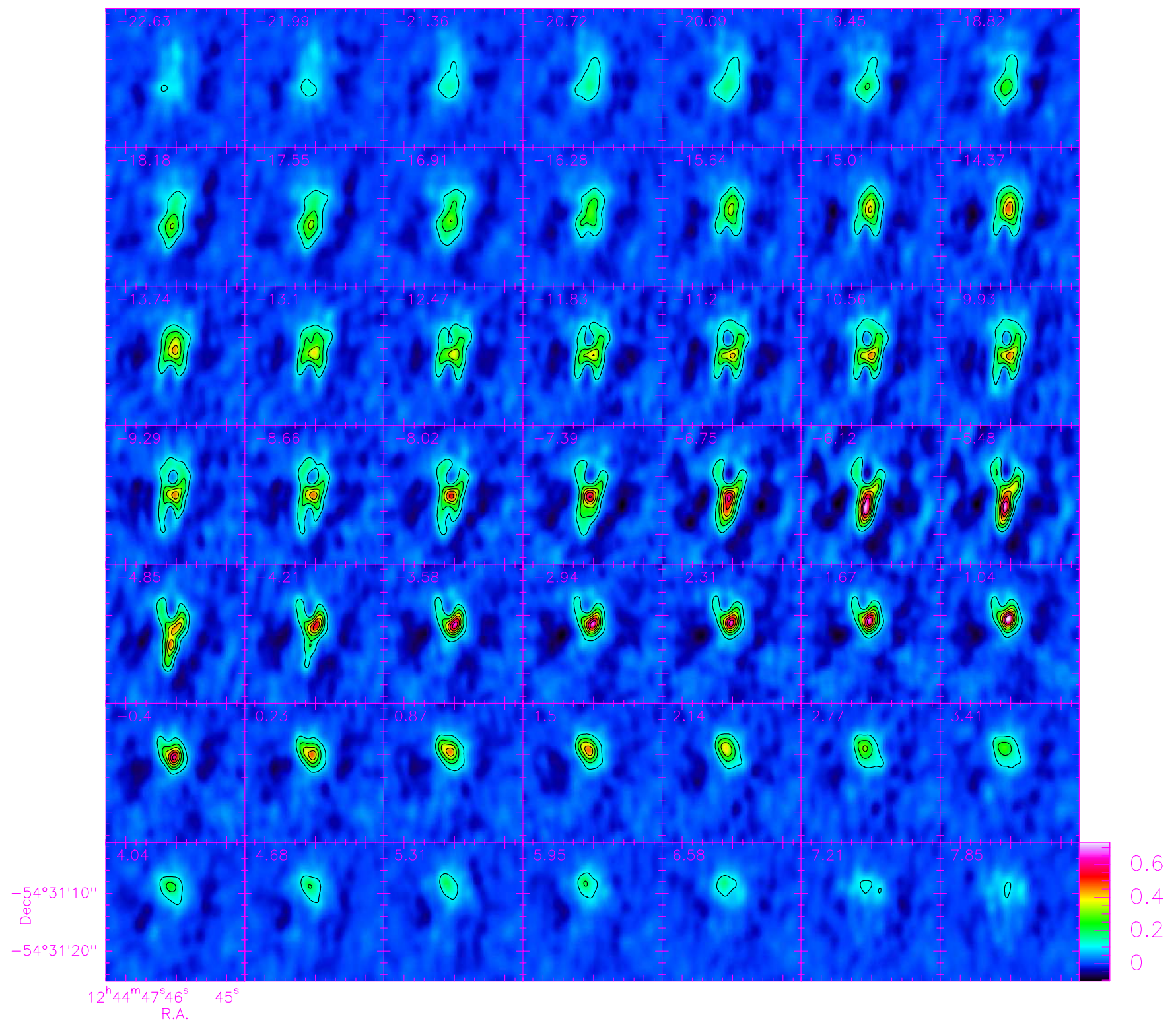

Figure 2. ALMA CO $(J=2-1)$ channel maps of the Boomerang, covering the radial velocity range $V_{\mathrm{lsr}}=-22.63$ to $7.85 \mathrm{~km} \mathrm{~s}^{-1}$. Contours levels are $0.1,0.2,0.3$, $0.4,0.5$, and $0.6 \mathrm{Jy}_{\text {beam }}{ }^{-1}$, and the color-coding of the intensity scale (in Jy beam ${ }^{-1}$ ) is shown at the bottom right corner. The central velocity for each panel is shown in its top left corner, and the channel width is $0.63 \mathrm{~km} \mathrm{~s}^{-1}$.
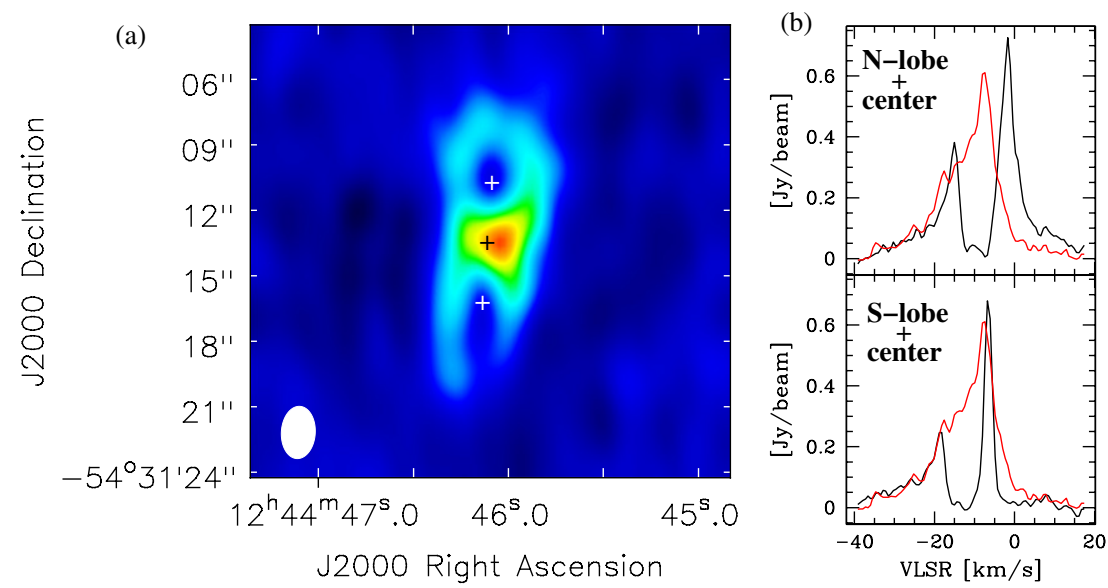

Figure 3. ALMA CO $(J=2-1)$ data of the Boomerang: (a) map at the systematic velocity (b) representative spectra. The latter have been extracted from the waist center (black cross) and the northern and southern lobes (white crosses). The waist spectrum is overlaid (in red) on each of the lobe spectra (in black) for comparison. 


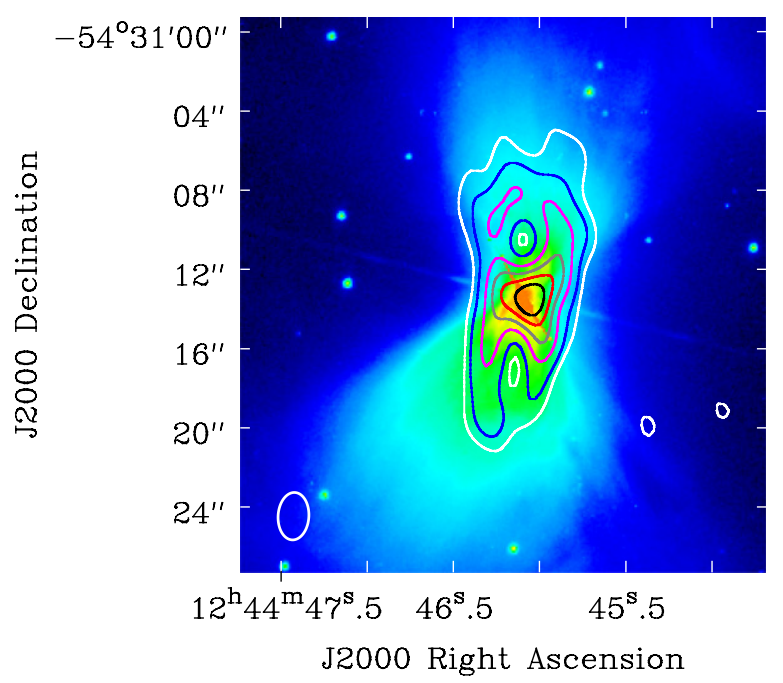

Figure 4. ALMA CO $(J=2-1)$ map (contours) of the Boomerang at the systemic velocity overlaid on a false-color $H S T$ /WFPC2 image taken with filter

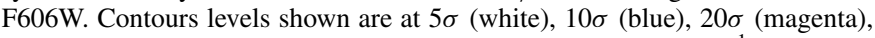
$30 \sigma$ (gray), $40 \sigma$ (red), and $50 \sigma$ (black), with $\sigma=7.5 \mathrm{mJy} \mathrm{beam}^{-1}$. The beam (FWHM) is shown as the white ellipse in the lower left corner.

emission extent is smaller than the angular scale at which the ALMA observations are expected to resolve out structures $\left(\gtrsim 17^{\prime \prime}\right)$, and consistent with this expectation, we find that the spatially integrated ALMA CO $(J=2-1)$ flux (3 Jy) is not significantly different from the single-dish (SEST) flux (3.1 Jy).

The long axis of the nebula seen in $\mathrm{CO}$ is roughly aligned with the symmetry axis of the nebula seen in the WFPC2 image (Figure 4). We find a detailed association between features in the $\mathrm{CO}(J=2-1)$ image and the HST image. Like the latter, the $\mathrm{CO}(J=2-1)$ map at the systemic velocity shows an hourglass structure. The $\mathrm{E}$ and $\mathrm{W}$ peripheries of this structure appear roughly cylindrical with an orientation that is consistent with that of the visible-light $\mathrm{S}$ lobe in several channels at and near the systemic velocity. However, at larger red-shifts relative to the systemic velocity, i.e., for $V_{\mathrm{lsr}} \gtrsim-5 \mathrm{~km} \mathrm{~s}^{-1}$, the P.A. appears to shift anti-clockwise, bringing it into rough alignment with the $\mathrm{W}$ limb of the visible-light $\mathrm{N}$ lobe. In summary, in spite of the large difference in the resolution of the visible-light and millimeterwave images that preclude a more precise comparison between the two, the ALMA data are consistent with the bulk of the CO emission arising from the walls of the lobe cavities seen via scattered-light in the HST image.

\subsubsection{Spatio-Kinematic Structure of the Lobes}

The position-velocity (PV) plot of the $\mathrm{CO}(J=2-1)$ emission for a cut along the major axis of the nebula (Figure 5) also reveals an hourglass-shaped structure, and shows the emission from the front wall (red-shifted) and back wall (blue-shifted) of the north and south lobes. The emission from each wall of the $\mathrm{S}$ lobe is blue-shifted relative to the corresponding wall of the northern lobe, confirming our inference that the southern (northern) lobe is tilted toward (away from) us. The tilt of the lobes inferred above is consistent with the larger overall visiblelight brightness of the southern lobe compared to the northern one-we expect the lobe closer to us to be brighter because for it, (1) the scattered light traverses a smaller column density of foreground circumstellar dust and (2) the starlight is scattered more efficiently toward the line-of-sight if the grains have a forward-peaked scattering phase function.

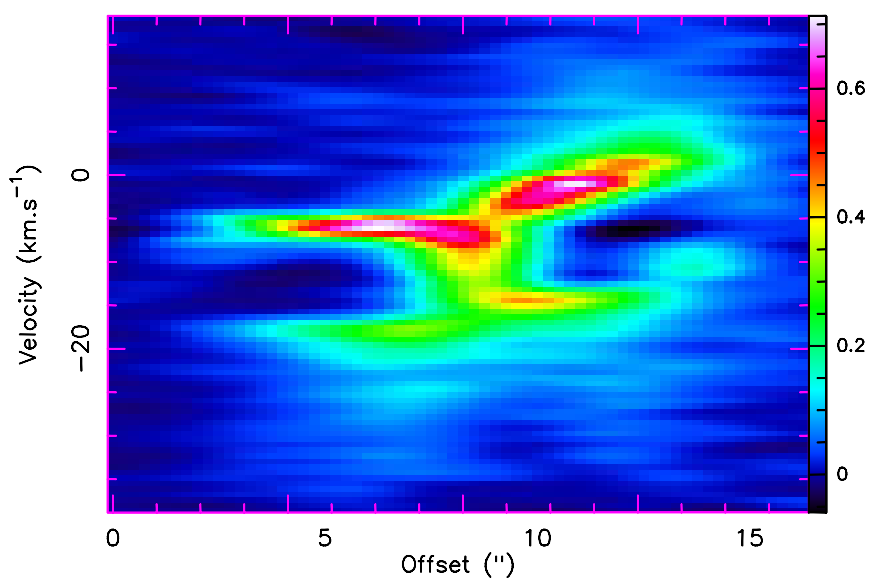

Figure 5. CO $(J=2-1)$ position-velocity diagram of intensity along the major axis of the nebula. Offsets are measured from south to north, beginning at R.A.= $12^{\mathrm{h}} 44^{\mathrm{m}} 46^{\mathrm{s}} .21$, decl. $=-54^{\circ} 31^{\prime} 22^{\prime \prime} .1$ and terminating at R.A. $=12^{\mathrm{h}} 44^{\mathrm{m}} 46^{\mathrm{s}} .02$, decl. $=-54^{\circ} 31^{\prime} 02^{\prime \prime}$. 4 . The color-coding of the intensity scale (in Jy beam ${ }^{-1}$ ) is shown on the right side of the panel.

Since all emission from the $\mathrm{S}$ lobe's far wall is red-shifted from the systemic velocity, we can set an upper limit of $15^{\circ}$ on the inclination of the nebula axis to the sky-plane, assuming radial expansion and a cylindrical geometry for the $\mathrm{S}$ lobe, using the $S$ lobe's diameter (3".3; as measured from the lateral separation of the mid-point of each wall in the CO $(J=2-1)$ image at the systemic velocity) and maximum projected length (6.'5; as measured for the red-shifted emission feature in the $\mathrm{S}$ lobe in Figure 5).

For similar velocity offsets from the systemic velocity, the red-shifted emission is, in general, significantly brighter than the corresponding blue-shifted one (e.g., Figure 5). This effect is most simply understood if (1) there is a temperature gradient across the thickness of the lobe walls such that the interior surface of the walls is hotter than the exterior one, and (2) the CO $(J=2-1)$ emission is optically thick. For optically thick emission at any given velocity offset, we can only see emission from the surface of each wall that is closest to us: hence, at red-shifted (blue-shifted) velocities, we see the hotter, interior (cooler, exterior) surfaces of the lobe walls.

We expect the lateral separation between the walls of each lobe in the channel maps to progressively decrease with increasing offset from the systemic velocity until they finally merge along the nebular axis (Figure 2). This "narrowing" effect is seen most clearly for the S lobe, where a prominent narrow linear feature appears at P.A. $\sim 175^{\circ}$ in the velocity range $V_{\mathrm{lsr}}=-7.39 \mathrm{~km} \mathrm{~s}^{-1}$ to $-4.21 \mathrm{~km} \mathrm{~s}^{-1}$, with a linear extent of about 5.9 as measured at the half-intensity points at $V_{\text {lsr }}=-5.48 \mathrm{~km} \mathrm{~s}^{-1}$. The blue-shifted equivalent of this feature for the $\mathrm{S}$ lobe, is seen in the velocity range $V_{\mathrm{lsr}}=-16.91 \mathrm{~km} \mathrm{~s}^{-1}$ to $-18.18 \mathrm{~km} \mathrm{~s}^{-1}$, but is fainter (since it comes from the exterior, cooler surface of the lobe) and less extended. The same "narrowing" effect can also be seen for the blue-shifted emission from the $\mathrm{N}$ lobe (e.g., at $V_{\mathrm{lsr}}=-16.28 \mathrm{~km} \mathrm{~s}^{-1}$ to $-19.45 \mathrm{~km} \mathrm{~s}^{-1}$ ), but the "narrowing" effect and the equivalent red-shifted feature is not seen for the far wall of the $\mathrm{N}$ lobe. The spatio-kinematic structure of the $\mathrm{N}$ lobe is clearly more complex than that of the S lobe.

\subsubsection{Spatio-Kinematic Structure of the Waist}

In a PV plot of a cut across the waist in the CO $(J=2-1)$ map (Figure 6), one can see evidence for a velocity-gradient 


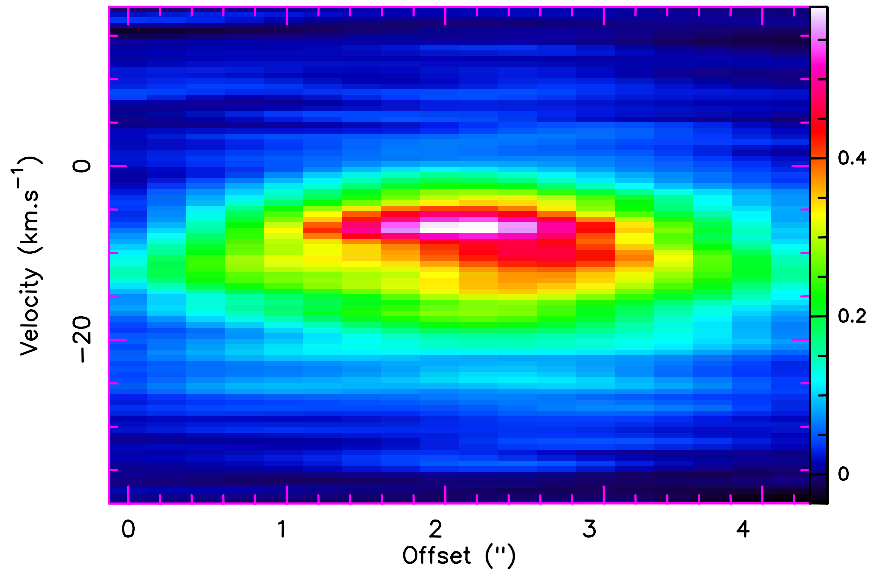

Figure 6. $\mathrm{CO}(J=2-1)$ position-velocity diagram of intensity along a cut traversing the waist of the Boomerang. Offsets are measured from east to west, beginning at R.A. $=12^{\mathrm{h}} 44^{\mathrm{m}} 46^{\mathrm{s}} .31$, decl. $=-54^{\circ} 31^{\prime} 12^{\prime \prime} .8$ and terminating at R.A. $=12^{\mathrm{h}} 44^{\mathrm{m}} 45^{\mathrm{s}} .81$, decl. $=-54^{\circ} 31^{\prime} 13^{\prime \prime} .4$. The color-coding of the intensity scale (in Jy beam ${ }^{-1}$ ) is shown on the right side of the panel.

in the line centroid, which appears to shift steadily from about $-7 \mathrm{~km} \mathrm{~s}^{-1}$ at an offset of about $\sim 1^{\prime \prime}$. 2 to $-11 \mathrm{~km} \mathrm{~s}^{-1}$ at an offset of $\sim 2$ ". 8 . In contrast, at low intensity levels, the emission is symmetric (green region in plot). The gradient appears to be real and not an artifact of the limited resolution mixing in emission from the $\mathrm{N}-\mathrm{S}$ outflow, as this would result in an even more pronounced gradient in the green region. However, the interpretation of the PV plot (e.g., as resulting from rotation) is not straightforward-e.g., the symmetric green outer region has its major axis at $-12 \mathrm{~km} \mathrm{~s}^{-1}$, but the mid-point of the asymmetric orange-red region in the center appears at $\sim-8 \mathrm{~km} \mathrm{~s}^{-1}$.

$$
\text { 3.3. } C O(J=1-0)
$$

The $J=1-0$ image (Figure 7 ) shows a central bipolar nebula in emission, roughly similar to that seen in the $J=2-1$ line, but due to the factor of two lower resolution at $2.6 \mathrm{~mm}$, the limb-brightened structure is not resolved. The bipolar nebula is surrounded on all sides by large patchy regions of absorption. The absorption regions lie roughly within a circle with a diameter of $\sim 50^{\prime \prime}$. Immediately beyond the absorption region, one can see faint patchy emission regions lying within a circle with a diameter of $\sim 65^{\prime \prime}$. The spectrum toward the center of the nebula (Figure 7(b)) shows a strong emission peak centered at the systemic velocity with some weak absorption features due to regions in the ultra-cold outflow that lie along the line-of-sight to the center. The FWZI of the emission component is about $85 \mathrm{~km} \mathrm{~s}^{-1}$, somewhat larger than that of the central emission component measured in the $\mathrm{CO}(J=2-1)$ line. In contrast, the spectrum (Figure 7(d)) averaged over the whole nebula shows, in addition to the central emission, absorption features extending to expansion velocities up to 170 (190) $\mathrm{km} \mathrm{s}^{-1}$ redward (blueward) of the systemic velocity.

The CO $(J=1-0)$ spectra toward the center of the nebula, extracted from the ALMA+SEST map (Figure 8), shows absorption over a wide range of velocities, different from the expectation of a shell expanding at a constant velocity, in which case the spectra would show absorption only at and near the outflow velocity. The spectra have been extracted from (and averaged over) two circular apertures, one with a diameter equal to the mean beam FWHM and the other with a diameter of twice the mean beam FWHM. If the absorption at relatively low outflow velocities was simply because of a projection effect, i.e., due to high-velocity material close to the center that is included within the aperture but is expanding at a large angle to the line-of-sight, then we would expect the intensity of the absorption feature in
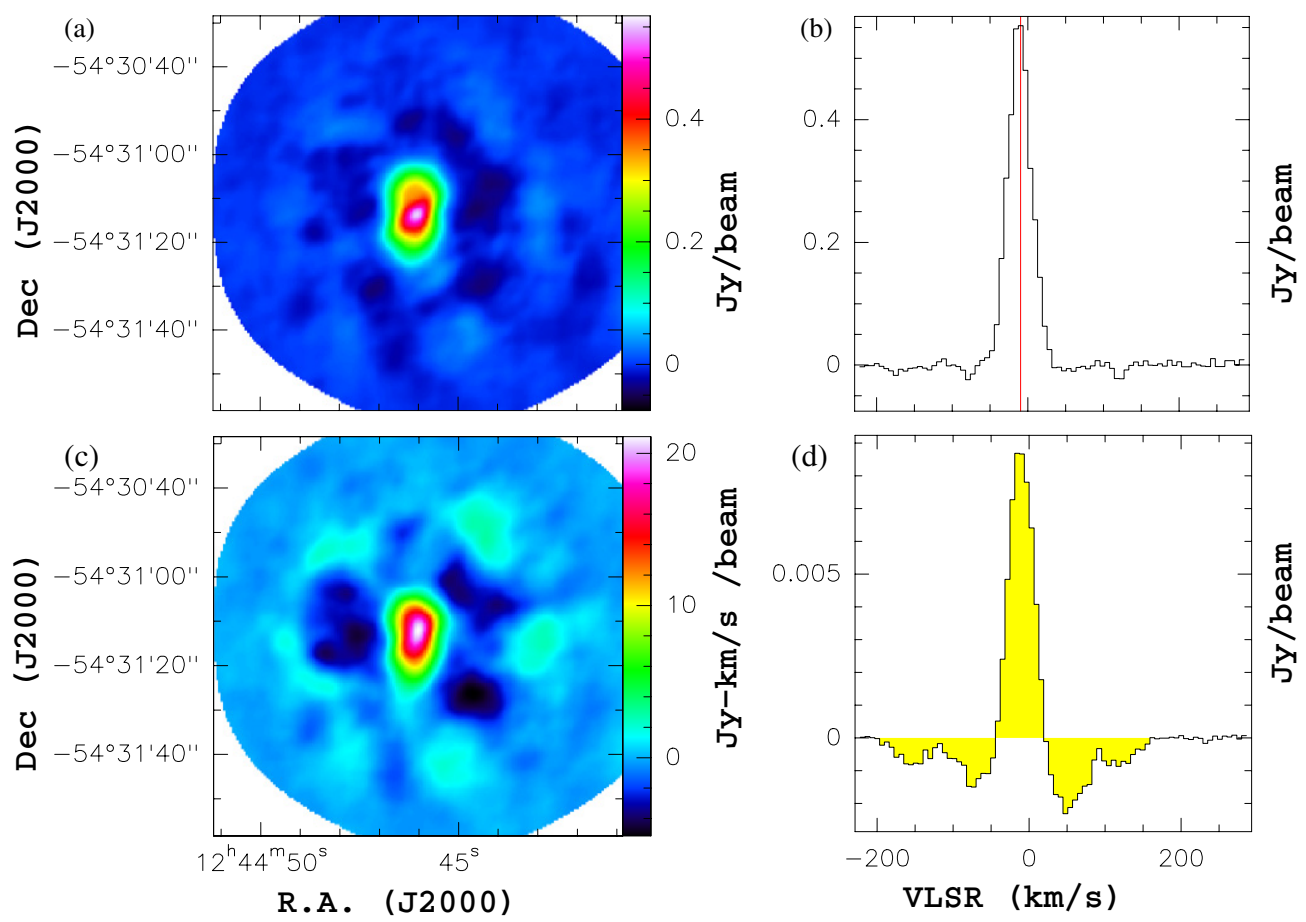

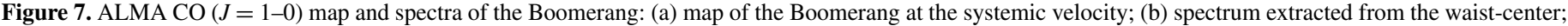

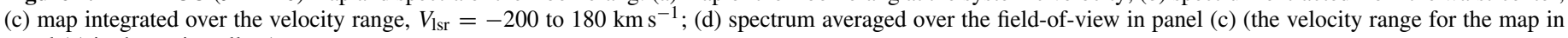
panel (c) is shown in yellow). 


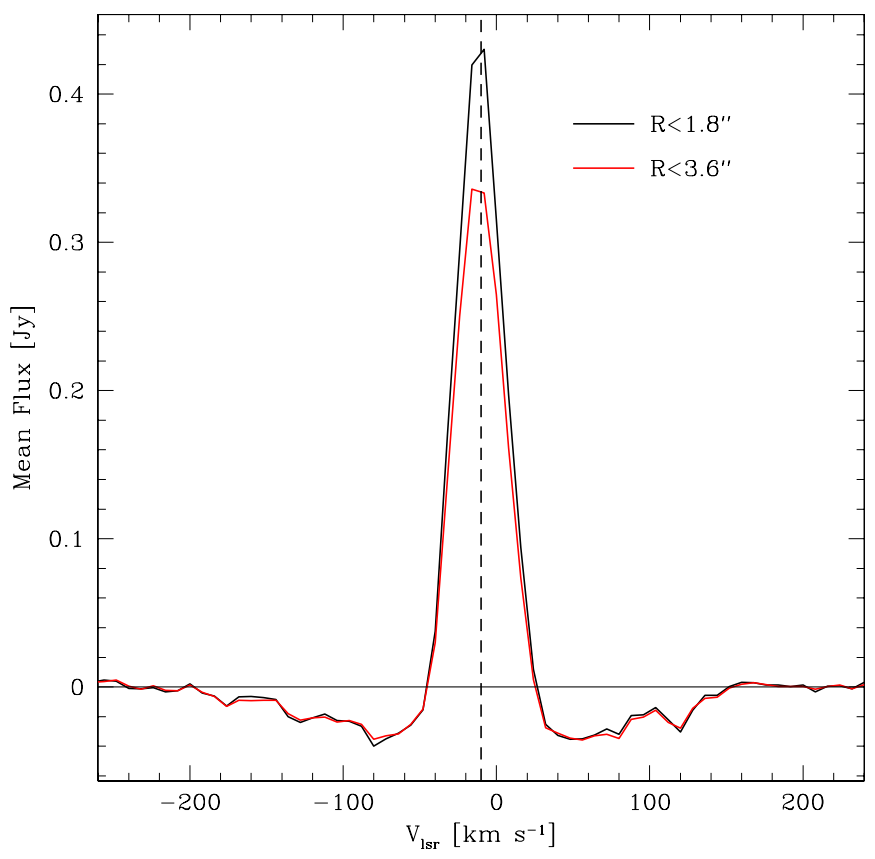

Figure 8. "Line-of-sight" CO $(J=1-0)$ spectra toward the center of the Boomerang, extracted from the ALMA+SEST map. The spectra have been extracted from (and averaged over) two circular apertures, one with a diameter equal to the mean beam FWHM and the other with a diameter twice the mean beam FWHM.

(A color version of this figure is available in the online journal.)

the larger-aperture spectrum to be significantly stronger than in the smaller-aperture one, contrary to what is observed. ${ }^{8}$

We expect a large fraction of the $\mathrm{CO}(J=1-0)$ absorbing cloud to be resolved out in our ALMA data because in the configuration used for our observations, the array was not sensitive to structure on scales $\gtrsim 35^{\prime \prime}$. As a result, we find that the absorption feature in the $\mathrm{CO}(J=1-0)$ spectrum, derived from spatially integrating over the full source, is much weaker in the ALMA map compared to the corresponding spectrum from the ALMA+SEST map (Figure 9).

However, we do not expect the central bipolar source seen in emission to be over-resolved, so the signal from this component should have the same intensity in both the ALMA and ALMA+SEST maps. This expectation is supported by our finding that the peak flux of the emission component in the ALMA spectrum ( $5 \mathrm{Jy}$ ) is only about $20 \%$ different from (larger than) that in the ALMA+SEST spectrum (as measured above a Gaussian fit to the underlying shape of the absorption component), a discrepancy that is well within the calibration uncertainties of both data sets.

\subsection{Continuum}

The continuum image at $1.3(2.6) \mathrm{mm}$, with a beam of $2^{\prime \prime} .2 \times 1^{\prime \prime} .1\left(4{ }^{\prime \prime} .1 \times 2\right.$.'.9) having its major-axis at P.A. $=-176.6$ (-33.9), shows a compact source, largely unresolved, with evidence for some extended weak emission (Figure 10). It is interesting that the $1.3 \mathrm{~mm}$ image shows a narrow linear feature extending about $3^{\prime \prime}$ south from the central peak, that, although of marginal significance, is aligned with the knotty, collimated feature seen in the HST image. The peak fluxes

\footnotetext{
8 The average emission feature is weaker for the larger-aperture because the central hourglass nebula becomes generally fainter with distance from the center.
}

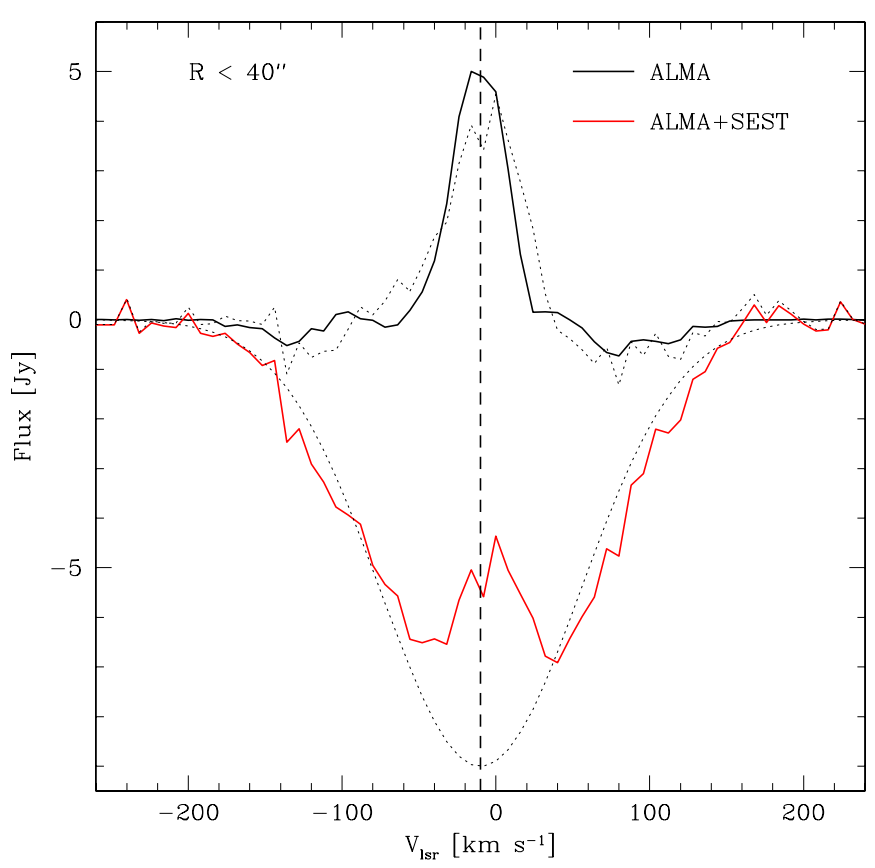

Figure 9. Spatially integrated $\mathrm{CO}(J=1-0)$ spectrum of the Boomerang over a circular aperture of radius $40^{\prime \prime}$ : (top) ALMA data only and (bottom) ALMA+SEST data. The dotted absorption curve is a Gaussian fit to the wings of the absorption feature and the dotted emission curve is the difference between the ALMA+SEST spectrum and the former.

(A color version of this figure is available in the online journal.)

extracted from the 1.3 and $2.6 \mathrm{~mm}$ continuum images, with the former convolved to the same beam as the latter, are $3.64 \mathrm{mJy}$ and $0.64 \mathrm{mJy}$, respectively.

The peak of the continuum emission, $\mathrm{Pk}_{m m}$, as measured in the 1.3 and $2.6 \mathrm{~mm}$ continuum images, has J2000 coordinates of R.A. $=12^{\mathrm{h}} 44^{\mathrm{m}} 45^{\mathrm{s}} .99$, decl. $=-54^{\circ} 31^{\prime} 13^{\prime \prime} .5$, with an error of about $\pm 00^{\prime \prime} 15$. This position is significantly offset from the phase-center. It is near to but slightly offset to the west (by about 0.9 ) from the location of the central point source in the Boomerang seen at near-IR to mid-IR wavelengths $\left(\mathrm{Pk}_{i r}\right)$, that has Two Micron All Sky Survey (2MASS) coordinates of R.A. $=12^{\mathrm{h}} 44^{\mathrm{m}} 46^{\mathrm{s}} .09$, decl. $=-54^{\circ} 31^{\prime} 13^{\prime \prime} .3$ (the DENIS, WISE, and Akari-Mid positions of $\mathrm{Pk}_{i r}$ are all within $\sim 0^{\prime \prime} 15$ of the 2MASS one). $\mathrm{Pk}_{m m}$ is also offset westward from the long-axis of the nebula seen in $\mathrm{CO}(J=2-1)$ by about $1^{\prime \prime}$. The brightness distribution of $\mathrm{CO}(J=2-1)$ in the waist is also not symmetric about the nebular axis, and its peak, at (and near) the systemic velocity is located at the same position as $\mathrm{Pk}_{m m}$ (within measurement errors).

Our ATCA data show no detectable emission at the position of the Boomerang Nebula with $1 \sigma$ upper limits of 23,34 , and $21 \mu \mathrm{Jy}$ beam $^{-1}$ at $5.5,9$, and $23 \mathrm{GHz}$, respectively.

\section{DISCUSSION}

The ALMA data validate some basic features of the SN97 model, which consists of two nested spherically symmetric shells: a warm inner shell extending $2.5-6$ " with an expansion velocity of about $35 \mathrm{~km} \mathrm{~s}^{-1}$ and a cool, extended outer shell extending $6^{\prime \prime}-33^{\prime \prime}$, with a velocity of about $164 \mathrm{~km} \mathrm{~s}^{-1}$. The latter shell is cooled below the temperature of the cosmic microwave background through adiabatic expansion. The ALMA observations show that the inner component is bipolar, with a dense waist, and the outer component is patchy but roughly circular 


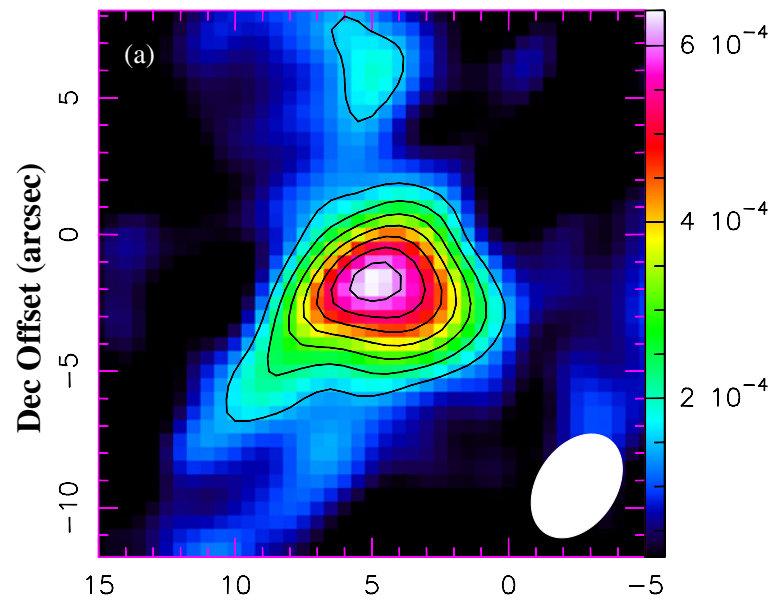

R.A. Offset (arcsec)

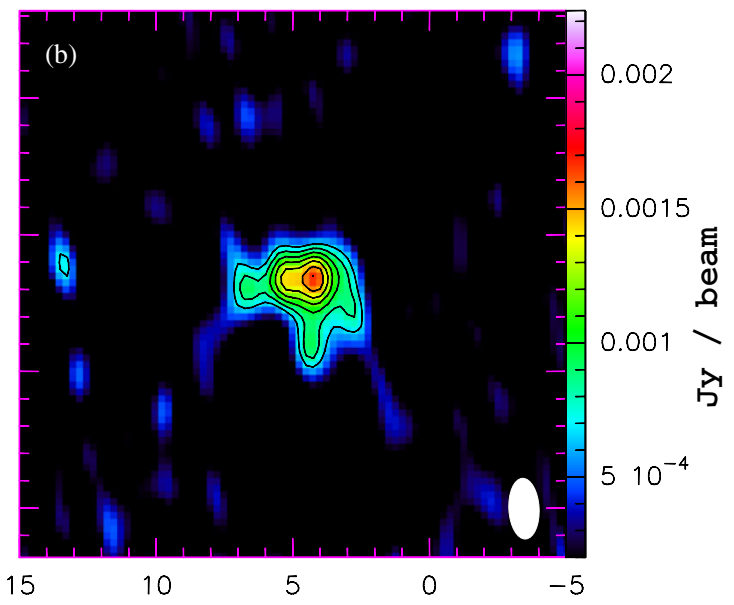

R.A. Offset (arcsec)

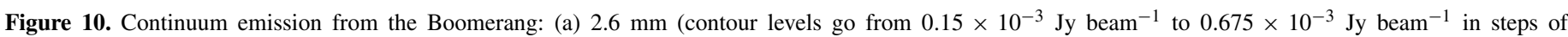

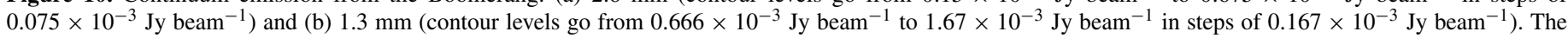
restoring beams are shown in the bottom right corner of each panel.

and similar in dimensions to the model, bearing in mind that a significant fraction of the flux in absorption has been resolved out.

Although SN97 adopted a constant expansion velocity for the ultra-cold outflow in their model, they admitted the possibility that the outflow velocity increased gradually from $35 \mathrm{~km} \mathrm{~s}^{-1}$ in the inner shell to $164 \mathrm{~km} \mathrm{~s}^{-1}$ in the outer one. Our ALMA data reveal a radially varying outflow velocity in the ultra-cold outflow, suggesting that the absorbing material in this outflow was either ejected with a velocity that has varied with time or in a single, "explosive" event with a wide distribution of velocities. In either case, adiabatic cooling due to expansion will still occur, but the temperature profile of SN97 with a constant velocity may need to be modified.

The good correspondence between the limb-brightened bipolar lobes seen in the ALMA CO $(J=2-1)$ and HST images confirms that these are hollow cavities with dense walls of molecular gas and dust producing both the molecular-line structures seen at millimeter wavelengths and the scattered-light structures seen at visible wavelengths. The molecular gas associated with the diffuse visible-light nebulosity surrounding the $\mathrm{N}$ and $\mathrm{S}$ lobes is not seen in the $\mathrm{CO}(J=2-1)$ map, presumably because it belongs to the ultra-cold outflow and does not emit in the $J=2-1$ line.

The diffuse biconical morphology seen in the visible is likely the result of preferential illumination of the ultra-cold outflow (that is, intrinsically spherical) due to the presence of a central dusty structure detected in the continuum images, which hinders the starlight from escaping at low latitudes (the long axis of the nebula defines the polar axis). Several radial streaks seen in the diffuse nebulosity provide support for this scenario, as these may then be simply explained as "shadows" due to inhomogeneous dust obscuration present around the central star. Fainter, smooth nebulosity is seen at low latitudes and is likely due to illumination of circumstellar material there by multiply scattered starlight.

The central, dusty structure that results in the preferential illumination of the ultra-cold outflow, may be a flared disk. Alternatively, since the opening angles of the diffuse nebulosity and the $\mathrm{V}$-shaped bases of the lobes, seen in the visible, appear to be similar, it is also plausible that the central dusty region was formed by heavy spherical mass-loss, but has a biconical physical cavity in it through which the starlight streams radially outwards. Such a cavity may have been carved out by a bipolar jet-e.g., for the PPN Hen 3-401, which also has cylindrical lobes with V-shaped bases. Sahai et al. (1999) concluded that the lobe shapes may be produced by a fast, momentum-conserving jet sweeping up material in an ambient medium with a radially decreasing density.

We suggest that a similar mechanism may have produced the central bipolar nebula in the Boomerang as well. This mechanism would require the jet velocity, $V_{\text {jet }}$, to be larger than the expansion velocity, $V_{\mathrm{amb}}$, of the ambient circumstellar material (i.e., the ultra-cold outflow in the case of the Boomerang), and the expansion velocity, $V_{\text {lob }}$, in the swept-up shell (i.e., the lobes) to be $<V_{\text {jet }}$ and $>V_{\text {amb }}$. This would have been a problem if the ultra-cold outflow had a radially constant outflow velocity of $164 \mathrm{~km} \mathrm{~s}^{-1}$ since the expansion velocity in the lobes is much lower. Thus our finding that the ultra-cold outflow is likely expanding at relatively low speeds in its inner regions is crucial for supporting the jet-interaction scenario that we have proposed for producing the central bipolar nebula in the Boomerang. The linear, knotty feature seen in the $H S T$ image (Figure 1, inset) may arise due to line emission (e.g., $\mathrm{H} \alpha$ ) from the inner regions of this jet. In order to test this idea, we will require spatially resolved spectroscopy of this feature and/or determination of proper motion in the knots showing high velocities using HST.

\subsection{Re-heating of the Ultra-cold Outflow: Photoelectric Dust Grain Heating}

Inspection of the patchy regions of weak emission in the outer regions of the ultra-cold shell in the ALMA CO $(J=$ 1-0) map and in the ALMA+SEST map shows that these patches, although brighter than their surroundings, remain in absorption against the microwave background. The reason they appear in emission in the ALMA data is because a significant part of the ultra-cool outflow absorption structure is smooth and resolved out by the interferometer. The presence of these regions can be a result of local variations (decreases) in column densities (thus producing less absorption) or warmer temperatures. However, the specific spatial distribution of these (i.e., generally at larger radii than the absorption patches) suggests that they are due to warmer temperatures because the 


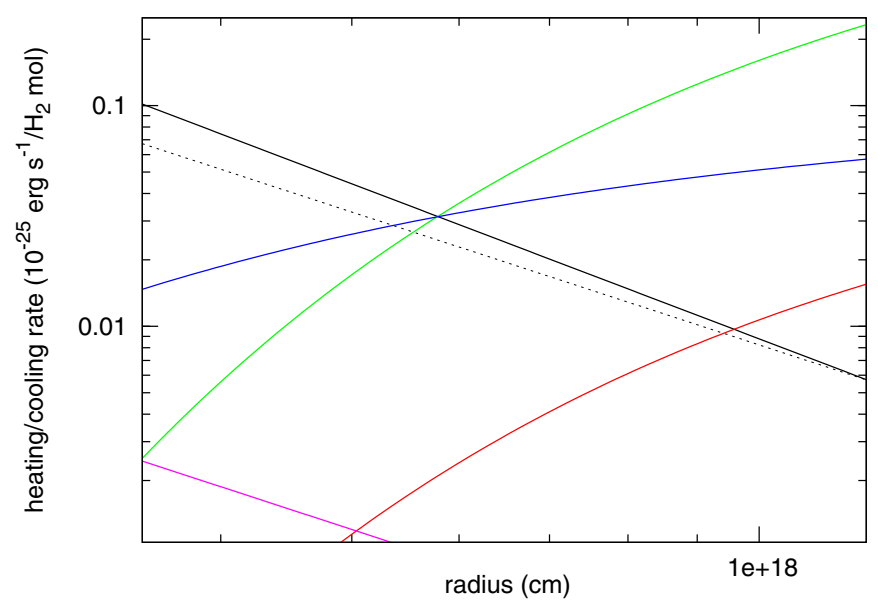

Figure 11. Adiabatic cooling rate ( $Q_{\text {adiab}}$, black curves), compared to various heating rates per $\mathrm{H}_{2}$ molecule, for the ultra-cold outflow in the Boomerang. The solid (dashed) black curve shows the adiabatic cooling rate for a constant (radially increasing) outflow velocity (see the text in Section 4 for details). The red and green curves show $Q_{p e}$ for a standard value of the unshielded photoelectric rate, $k_{p e}=10^{-26} \mathrm{erg} \mathrm{s}^{-1}$, and a much larger value, $1.5 \times$ $10^{-25} \mathrm{erg} \mathrm{s}^{-1}$, respectively, and the magenta curve shows the dust-gas frictional heating rate; for each of these, the dust-to-gas ratio is $1 / 200$. The blue curve shows $Q_{p e}$ for a dust-to-gas ratio lower by a factor of 3.3, and the standard $k_{p e}$.

ultra-cold outflow is expected to ultimately begin heating up due to heating via grain photoelectric heating followed by heating due to photodissociation of CO (e.g., Huggins et al. 1988). The latter is not expected to be a significant contributor at radii smaller than the photodissociation radius of the self-shielded $\mathrm{CO}$ in the outer shell, about $5 \times 10^{18} \mathrm{~cm}$ (Knapp \& Chang 1985).

We can roughly estimate the radius, $r_{p e}$, at which grain photoelectric heating starts becoming effective by equating the adiabatic cooling rate per $\mathrm{H}_{2}$ molecule, $Q_{\text {adiab }}=$ $2 k_{B} V_{\exp } T_{\text {kin }}(r) / r$, with the photoelectric heating rate, $Q_{p e}=$ $k_{p e} \exp [-\tau(1000 \AA)]$, where $k_{B}$ is the Boltzmann constant, $V_{\exp }$ is the outflow velocity, $T_{\text {kin }}$ is the kinetic temperature, $k_{p e}$ is the unshielded heating rate (in $\left.\operatorname{erg~s}^{-1}\right)$, and $\tau(1000 \AA$ ) is the circumstellar dust optical depth to the interstellar UV radiation field at a nominal wavelength of $1000 \AA$. Substituting $T_{\text {kin }}(r)=2.8 K\left(-r / 1.35 \times 10^{17}\right)^{-4 / 3}(\mathrm{SN} 97)$, and $\tau(1000 \AA)=$ $3.44 \times 10^{21} \dot{M}(100 / \delta) / r$ (Morris \& Jura 1983), with the gas-todust ratio, $\delta=200$, and $\dot{M}=1.3 \times 10^{-3} M_{\odot} \mathrm{yr}^{-1}$, we compare $Q_{p e}(r)$ to $Q_{\text {adiab }}$, for $k_{p e}=10^{-26}$ (e.g., Huggins et al. 1988), and find that $r_{p e} \sim 10^{18} \mathrm{~cm}$ (Figure 11), larger than the observed value $\left(\sim 25^{\prime \prime}\right.$, or $\left.0.56 \times 10^{18} \mathrm{~cm}\right)$ by almost a factor of two. This could be accommodated, e.g., by an increase in $k_{p e}$ by a factor of $\sim 15$, or an increase in the gas-to-dust ratio by a factor of $\sim 3$ (Figure 11). Given the uncertainties in the envelope parameters and the dust grain properties of such a high-velocity outflow (e.g., very small grains can lead to values of $k_{p e}$ as high as $10^{-25}$, Jura 1976), external photo-electric heating is clearly a viable mechanism for the outer emission. A detailed calculation would also need to take into account the clumpy structure observed in the gas.

The above discussion is based on a constant expansion velocity, but the $\mathrm{CO}(J=1-0)$ observations (Section 3.3) suggest that there is a range of velocities present in the outflows. In order to investigate how this might affect the cooling, we assume that the expansion velocity of the ultra-cold outflow varies as a power-law, $V^{\prime}(r)=V_{o}\left(r / r_{o}\right)^{\alpha} \mathrm{km} \mathrm{s}^{-1}$, and analytically re-derive the radial dependence of the kinetic temperature and adiabatic cooling. With $Q_{\text {adiab }}^{\prime}=2 k_{B} V(r) T_{\text {kin }}(r)(1+$ $1 / 2 d \ln V / d \ln r) / r$ (e.g., Goldreich \& Scoville 1976), and assumed to dominate the heating-cooling terms, ${ }^{9}$ we find that $T_{\text {kin }}^{\prime}(r)=T_{i}\left(r / r_{i}\right)^{-(4+2 \alpha) / 3}$ (i.e., the kinetic temperature falls more steeply with radius than in the constant expansion velocity case). A determination of $T_{0}$ will require a new radiativetransfer/thermodynamic model to fit the SEST single-dish data (as in SN97), is outside the scope of this paper (and deferred to after we obtain Cycle 1 data). However, since the average kinetic temperature in the ultra-cold outflow is constrained by the observed absorption, we estimate $T_{0}$ by making the simple assumption that $T_{\text {kin }}^{\prime}(r)$ at some nominal average radius, $r_{a v}$, is the same as that for the constant expansion case. We set $V^{\prime}(r)=164 \mathrm{~km} \mathrm{~s}^{-1}\left(r / r_{o}\right)^{\alpha}$, with $\alpha=1$, and take $r_{a v}$ to be the geometric average of the inner and outer radii of the ultra-cold outflow in the SN97 model (i.e., $r_{i}=6^{\prime \prime}$ and $r_{o}=33^{\prime \prime}$, respectively), giving $T_{0}=4.9 \mathrm{~K}$. The resulting radial variation of $Q_{\text {adiab }}^{\prime}$, shown in Figure 11, is not very different from $Q_{\text {adiab }}$.

Finally, we note that since an outflow with a mass-loss rate that is constant with time and a radially increasing outflow velocity offers less optical depth to the interstellar UV radiation field in its outer regions compared to one where the outflow velocity is constant, the discrepancy that we found between the observed and estimated values of $r_{p e}$ is likely to be smaller.

\subsection{Thermal Dust Emission from the Waist}

We fit the ratio of the peak 1.3 and $2.6 \mathrm{~mm}$ continuum fluxes, measured within the same beam of size 4 ". $1 \times 2$ ".9 (see Section 3.4), $R=5.7$ (although signal-to-noise ratio is modest and $1.3 \mathrm{~mm}$ UV coverage is still sparse) as follows. Assuming a power-law dust emissivity, i.e., $\kappa(v) \propto v^{\beta}$, we find that $\beta=0.5$ in the Rayleigh-Jeans (R-J) limit, since $R\left(\lambda_{1} / \lambda_{2}\right)=\left(\lambda_{1} / \lambda_{2}\right)^{(2+\beta)}$. If we drop the R-J approximation, then determining $\beta$ requires a knowledge of the dust temperature, $T_{d}$. Taking $\beta=0.6,1,1.5$, we get $T_{d}=45 \mathrm{~K}$, $9.5 \mathrm{~K}, 5.0 \mathrm{~K}$ and $r_{d}=1^{\prime \prime} .9,236^{\prime \prime}, 6800^{\prime \prime}$, respectively, using $r_{d}=\left(L_{*} T_{*}^{\beta} / 16 \pi \sigma\right)^{1 / 2} T_{d}^{-(2+\beta / 2)}$, where $L_{*}$ and $T_{*}$ are the stellar luminosity and temperature (e.g., Herman et al. 1986). Since the continuum emission arises from a compact source with an observed value of $r_{d} \sim 2^{\prime \prime}$, we conclude that $\beta \sim 0.6$ and $T_{d} \sim 45 \mathrm{~K}$ (see Figure 12). Realistically, extinction and reddening of starlight needs to be considered, allowing somewhat higher $\beta$ and lower $T_{d}$ values; e.g., if only $10 \%$ of the total stellar flux (reddened to $900 \mathrm{~K}$ ) is available for heating the dust, then $\beta \sim 0.7$ and $T_{d} \sim 23 \mathrm{~K}$. The low value of $\beta$ suggests that the grains in the waist producing the observed emission are several millimeters in size (e.g., Draine 2006). The radio continuum observations at 5.5, 9, and $23 \mathrm{GHz}$ show no source, so free-free emission is unlikely to contribute to the 1.3 and $2.6 \mathrm{~mm}$ fluxes (Figure 12).

There is now a growing body of observational evidence for such large grains in the dusty equatorial regions of post-AGB objects (e.g., see Sahai et al. 2011a) that has opened up an exciting new opportunity to probe the very early stages of grain coagulation, i.e., on very short time-scales (i.e., 1000 yr), which is not possible with studies of planet-forming disks, since the latter are typically $\gtrsim 10^{6}$ yr old (Sahai 2009).

\footnotetext{
9 The assumption is quite reasonable since SN97 showed that the dust frictional heating term is much smaller, by a factor of $\sim 50$ at $r=10^{17} \mathrm{~cm}$, in the constant outflow velocity model.
} 


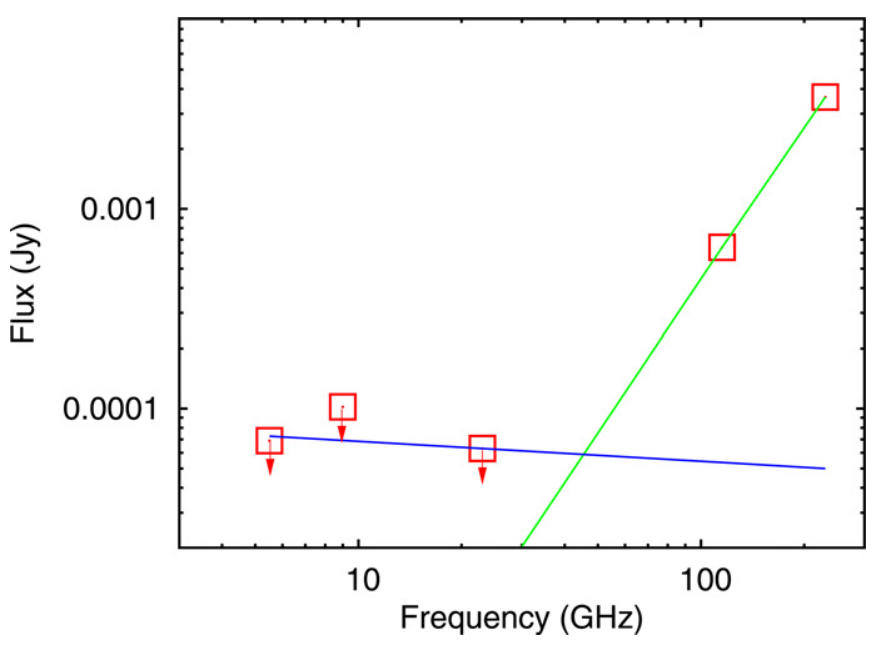

Figure 12. ALMA millimeter-wave continuum fluxes for the central source and $3 \sigma$ upper limits from ATCA. The data (red squares) have been fitted with a two-component model consisting of optically thin (1) thermal dust emission from millimeter-sized grains (thus with a shallow emissivity power-law index 0.6 ) at a temperature of $45 \mathrm{~K}$ (green curve) and (2) an upper limit to the free-free emission (blue curve).

(A color version of this figure is available in the online journal.)

The mass of dust in the central source derived from the above fluxes and taking $T_{d}=45 \mathrm{~K}$, is $M_{d} \sim 3.5 \times 10^{-4} M_{\odot}$, and the total mass is $M \sim 0.071 M_{\odot}$, assuming a dust opacity of $\kappa(1.3 \mathrm{~mm}) \sim 1.5 \mathrm{~cm}^{2} \mathrm{~g}^{-1}$ and a gas-to-dust ratio of $\delta=200$. The expansion time scale for the dust-continuum emitting region, assuming its size to be the geometric average of the major and minor axis of the elliptical beam used to measure the dust continuum $\left(3^{\prime \prime} .45\right.$ or $\left.7.76 \times 10^{16} \mathrm{~cm}\right)$ and an expansion velocity equal to half the FWZI of the $\mathrm{CO}(J=2-1)$ line profile of the waist, $32.5 \mathrm{~km} \mathrm{~s}^{-1}$, is about $400 \mathrm{yr}$, implying a mass-loss rate of $\sim 1.8 \times 10^{-4} M_{\odot} \mathrm{yr}^{-1}$. This rate is comparable to that derived by SN97 for the inner shell from the single-dish $\mathrm{CO}$ data, $\gtrsim 10^{-4} M_{\odot} \mathrm{yr}^{-1}$.

\section{CONCLUSIONS}

We have obtained ALMA maps of millimeter-wave line and continuum emission at the highest angular resolution to date of the Boomerang Nebula, the coldest known object in the universe. We compare these to high-resolution imaging at $0.6 \mu \mathrm{m}$ obtained with $H S T$. Our principal conclusions are as follows.

1. The Boomerang Nebula consists of a central, expanding hourglass-shaped lobe structure with a dense waist that is seen in emission in $\mathrm{CO}(J=2-1)$, surrounded by a patchy, ultra-cold outflow seen in absorption against the microwave background in the $\mathrm{CO}(J=1-0)$ line.

2. The HST imaging reveals the precise geometrical shapes of the lobes, which flare out from the center in a wide $\mathrm{V}$ shape, and then follow a roughly cylindrical shape. Each limb-brightened lobe is embedded in a larger, more diffuse conical nebulosity with an opening angle that is the same as that of its wide V-shaped inner region.

3. There is good correspondence between the limb-brightened hourglass lobes seen in $\mathrm{CO}(J=2-1)$ and the visiblelight imaging, showing that these are hollow cavities with dense walls of molecular gas and dust. The diffuse biconical nebulosity seen in the visible wavelength range surrounding these limb-brightened lobes is likely the result of preferential illumination of the ultra-cold outflow by light from the central star due to the presence of the central dusty structure seen in the continuum images.

4. The ratio of the $1.3 \mathrm{~mm}$ to the $2.6 \mathrm{~mm}$ continuum flux, $R=5.7$, is low compared to the expectation from thermal emission by small grains. Radio observations at 5.5, 9, and $23 \mathrm{GHz}$ show no source, so free-free emission does not contribute to the millimeter-wave fluxes. We infer a dust temperature of $\sim 45 \mathrm{~K}$ and a power-law dust emissivity exponent $\beta \sim 0.6$; the low value of $\beta$ suggests that the grains in the waist producing the observed emission are several millimeters in size.

5. The total mass associated with the dusty waist is $M \sim$ $0.071 M_{\odot}$ (assuming a gas-to-dust ratio of $\delta=200$ and a dust opacity of $1.5 \mathrm{~cm}^{2} \mathrm{~g}^{-1}$ at $1.3 \mathrm{~mm}$ ). The expansion time scale for the dust-continuum emitting region is about $400 \mathrm{yr}$, implying a mass-loss rate of $\sim 1.8 \times 10^{-4} M_{\odot} \mathrm{yr}^{-1}$.

6. A comparison of the single-dish and ALMA CO $(J=1-0)$ map shows that a large fraction of the absorption signal from the ultra-cold outflow is resolved out in our ALMA CO $(J=1-0)$ map. The observed absorption regions are seen to extend over a region $\sim 50^{\prime \prime}$ in size, beyond which can be seen faint patchy "emission" regions, lying within a region of size $\sim 65^{\prime \prime}$.

7. The combined ALMA+SEST map shows that the outer patchy "emission" regions, although brighter than their surroundings, remain in absorption against the microwave background; the presence of these regions is consistent with the re-warming of the ultra-cold gas due to photoelectric grain heating.

8. The $\mathrm{CO}(J=1-0)$ spectra toward the center of the nebula, extracted from the ALMA+SEST map, shows absorption over a wide range of velocities, implying that the absorbing material in the ultra-cold outflow was either ejected with a velocity that has varied with time, or in a single, explosive event with a wide distribution of velocities.

9. The central bipolar nebula is likely the result of a fast, momentum-conserving bipolar jet interacting with the relatively slowly expanding material present in the inner regions of the ultra-cold outflow. This jet may also be responsible for carving out biconical cavities in a central dusty structure through which the starlight streams out preferentially to produce the large-scale diffuse biconical appearance of the Boomerang Nebula. Plausible evidence for such a jet is provided by the presence of a linear, knotty feature emanating from the central star, seen in the HST image.

This paper makes use of the following ALMA data: ADS/JAO.ALMA\#2011.0.00510.S. ALMA is a partnership of ESO (representing its member states), NSF (USA), and NINS (Japan), together with NRC (Canada), NSC, and ASIAA (Taiwan), in cooperation with the Republic of Chile. The Joint ALMA Observatory is operated by ESO, AUI/NRAO, and NAOJ. The National Radio Astronomy Observatory is a facility of the National Science Foundation operated under cooperative agreement by Associated Universities, Inc. R.S.'s contribution to the research described here was carried out at JPL, California Institute of Technology, under a contract with NASA and partially funded through the internal Research and Technology Development program. W.V. acknowledges support by the Deutsche Forschungsgemeinschaft (DFG; through the Emmy Noether Research grant VL 61/3-1) and Marie Curie Career 
Integration Grant 321691. This work was supported in part by NSF grant AST 08-06910 (to P.J.H.).

\section{REFERENCES}

Draine, B. T. 2006, ApJ, 636, 1114

Goldreich, P., \& Scoville, N. 1976, ApJ, 205, 144

Herman, J., Burger, J. H., \& Penninx, W. H. 1986, A\&A, 167, 247

Huggins, P. J., Olofsson, H., \& Johansson, L. E. B. 1988, ApJ, 332, 1009

Jura, M. 1976, ApJ, 204, 12

Knapp, G. R., \& Chang, K. M. 1985, ApJ, 293, 281
Morris, M., \& Jura, M. 1983, ApJ, 264, 546

Sahai, R. 2009, astro2010: The Astronomy and Astrophysics Decadal Survey, 2010 (Washington, DC: National Academies), http://sites.nationalacademies. org/bpa/BPA_050603

Sahai, R., Bujarrabal, V., \& Zijlstra, A. 1999, ApJL, 518, L115

Sahai, R., Claussen, M. J., Schnee, S., et al. 2011a, ApJL, 739, L3

Sahai, R., Morris, M., Sánchez Contreras, C., \& Claussen, M. 2007, AJ, 134, 2200

Sahai, R., Morris, M. R., \& Villar, G. G. 2011b, AJ, 141, 134

Sahai, R., \& Nyman, L.-Å. 1997, ApJL, 487, L155 (SN97)

Wegner, G., \& Glass, I. S. 1979, MNRAS, 188, 327 